\title{
Bile duct preserving pancreatic head resection (BDPPHR): Can we conclusively define the extent of head resection in surgery for chronic pancreatitis?
}

\author{
Pagadala Naga Balaji Nitesh and Biju Pottakkat \\ Department of Surgical Gastroenterology, Jawaharlal Institute of \\ Post Graduate Medical Education and Research (JIPMER), Pondicherry, India
}

\begin{abstract}
The role of surgical management for chronic pancreatitis in providing pain relief and improving quality of life is significant. Surgical techniques involving pancreatic head resection scored more over the drainage procedures. Among the resection procedures, Frey's procedure received widespread acceptance. However, the exact extent of pancreatic head resection to be performed and the limits of the resection are still debatable. The present report of bile duct preserving pancreatic head resection (BDPPHR) describes an innovative approach to the pancreatic head and conclusively defines the extent of head resection. The simplicity, feasibility and easy reproducibility of the BDPPHR is also reiterated. (Ann Hepatobiliary Pancreat Surg 2020;24:309-313)
\end{abstract}

Key Words: Chronic pancreatitis; Surgery; Pancreatic head resection; Posterior pancreatic capsule

\section{INTRODUCTION}

The concept of early surgical management for chronic pancreatitis (CP) has significant impact on prevention of deterioration in the quality of life of patients. Resection procedures are associated with long-lasting pain relief and will prevent further complications associated with chronic pancreatitis, when compared to drainage procedures. In view of associated morbidity, extensive resection procedures like total pancreatectomy should be avoided in patients with disease process localised to head of pancreas. Currently, Frey's procedure, Beger procedure and Whipple pancreaticoduodenectomy are the common resection procedures performed in patients with CP. In spite of significantly increased acceptance of the Frey's procedure, the debate continues regarding the exact extent of resection of head of pancreas performed in this procedure. Due to varied reasons, the original Frey's procedure described in 1987 has been modified in the context of extent of head resection into modified Frey's and minimum
Frey's procedure. ${ }^{1-3}$ Amount of diseased pancreatic head tissue retained after these modified procedures is a matter of concern. To standardise the resection procedure for $\mathrm{CP}$, we describe a technique of bile duct preserving pancreatic head resection (BDPPHR).

\section{SURGICAL TECHNIQUE}

The abdominal cavity is usually exposed through bilateral subcostal incision with the use of self-retaining retractor. A complete Kocher maneuver till the identification of superior mesenteric vein (SMV) is performed with the intent of manual palpation of gland for assessment of consistency. This mobilisation of head of pancreas also aids during the coring of head of pancreas to secure hemostasis with palpating hand beneath the head of pancreas. We recommend the plane of kocherisation to be slightly away from the duodenum in order to avoid any breach in the posterior pancreatic capsule. The anterior surface of entire pancreas is exposed by releasing the gas-

Received: January 19, 2020; Revised: April 10, 2020; Accepted: April 12, 2020

Corresponding author: Biju Pottakkat

Department of Surgical Gastroenterology, Jawaharlal Institute of Post Graduate Medical Education and Research (JIPMER), Pondicherry 605006, India

Tel: +91-7598566982, Fax: +91-8179705152, E-mail: bijupottakkat@gmail.com

Copyright (C) 2020 by The Korean Association of Hepato-Biliary-Pancreatic Surgery

This is an Open Access article distributed under the terms of the Creative Commons Attribution Non-Commercial License (http://creativecommons.org/ licenses/by-nc/4.0) which permits unrestricted non-commercial use, distribution, and reproduction in any medium, provided the original work is properly cited. Annals of Hepato-Biliary-Pancreatic Surgery • pISSN: 2508-5778 - eISSN: 2508-5859 
trocolic ligament till the splenic flexure of colon and thereby approaching the lesser sac. Any adhesions between posterior aspect of stomach and anterior surface of pancreas needs to be released. The right gastroepiploic vessels are ligated and divided at the superior border of head of the pancreas to provide complete exposure of anterior surface of the head and neck of the pancreas. No further dissection is warranted at the superior border of the pancreas. Meticulous dissection is performed along the anterior and right lateral surface of SMV to provide optimal exposure of uncinate process. It facilitates complete coring of uncinate process of pancreas with SMV in view and also prevents any inadvertent injury to SMV. This might require even ligation of the draining veins from uncinate process to SMV. We never initiate pancreatic head resection before complete delineation of SMV as a precaution to avoid SMV injury. The inferior border of body and tail of the pancreas is mobilised.

Dilated pancreatic duct is often palpated as groove along the axis of the gland. In case of difficulty with ductal identification by palpation, we routinely perform intraoperative ultrasound for the identification of pancreatic duct. Exposure of pancreatic duct is ensured with 21gauge needle with syringe placed in duct and incising the anterior pancreatic tissue over the needle using electrocoagulation. Ductal probe or fine right-angled clamp is used to probe the direction of the duct. Pancreatic duct is exposed till distally within $0.5 \mathrm{~cm}$ of the end of the tail of the pancreas. Proximally in the head of pancreas, pancreatic tissue anterior to duct of Wirsung is incised till within $0.5 \mathrm{~cm}$ of the ampulla of Vater. The pancreatic duct patency near the ampulla of Vater is assessed by passing $8 \mathrm{Fr}$ infant feeding tube through the ampulla and palpating the tube within the duodenal lumen. All the ductal calculi, including branch duct calculi, identified during ductal exposure are removed thoroughly.

We recommend resection of head of the pancreas with posterior pancreatic capsule as the limit of resection. In our observation, posterior pancreatic capsule is thickened and is a tough structure in patients with chronic pancreatitis due to inflammation. Attempts to delineate posterior pancreatic capsule is made by blunt dissection through the anterior surface pancreatic parenchyma as shown in Fig. 1 and also by graded progression of resection of slices of pancreatic head using electrocoagu- lation. BDPPHR begins by excising pancreatic parenchyma in the uncinate process of pancreas in a plane perpendicular to the surface of pancreatic parenchyma as the first step. Once posterior pancreatic capsule is reached through perpendicular plane of pancreatic parenchymal resection at uncinate process, then slices of pancreatic parenchyma can be easily stripped off posterior pancreatic capsule from below upwards with the help of electrocoagulation. After excising each slice of pancreatic tissue, the remaining amount of pancreatic tissue needed to be removed is accurately assessed and proceeded. With progressively proceeding the resection in a similar manner, complete pancreatic head resection can be accomplished. In this technique, authors want to reiterate that resection proceeds in plane almost perpendicular to the surface of pancreas and the entire pancreatic parenchymal tissue including ducts of Wirsung, ducts of Santorini and its branch ducts in the head of pancreas, beyond its posterior wall till the posterior pancreatic capsule, are excised as shown in Fig. 2.

The right lateral extent of resection is limited by leaving a $0.5 \mathrm{~cm}$ rim of pancreatic parenchyma in order to preserve pancreaticoduodenal vascular arcade. Since the bile duct is in the right superolateral part of pancreatic head resection, we specifically focus in this technique to

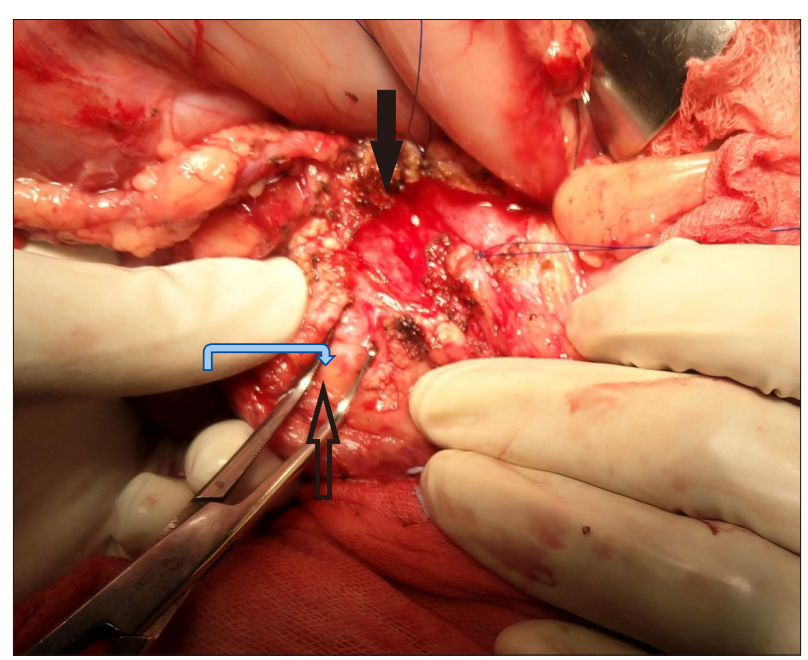

Fig. 1. Image showing technique of blunt dissection through anterior surface of the pancreatic parenchyma with the attempt to define posterior pancreatic capsule in head of the pancreas (marked with transparent arrow). Entire pancreatic duct in the body and tail region of pancreas was exposed (pointed with Black arrow). The Left hand of operating surgeon behind the kocherised duodenum and head of pancreas is guiding the procedure. Exposed posterior pancreatic capsule is marked with blue arrow. 


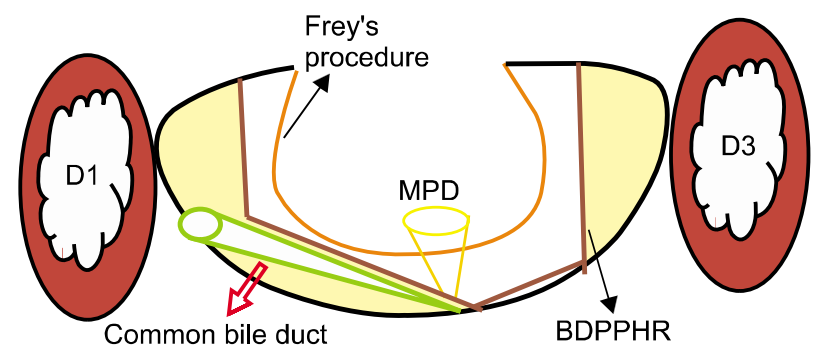

Fig. 2. Line diagram showing perpendicular plane of transection in BDPPHR as compared with Frey's procedure.

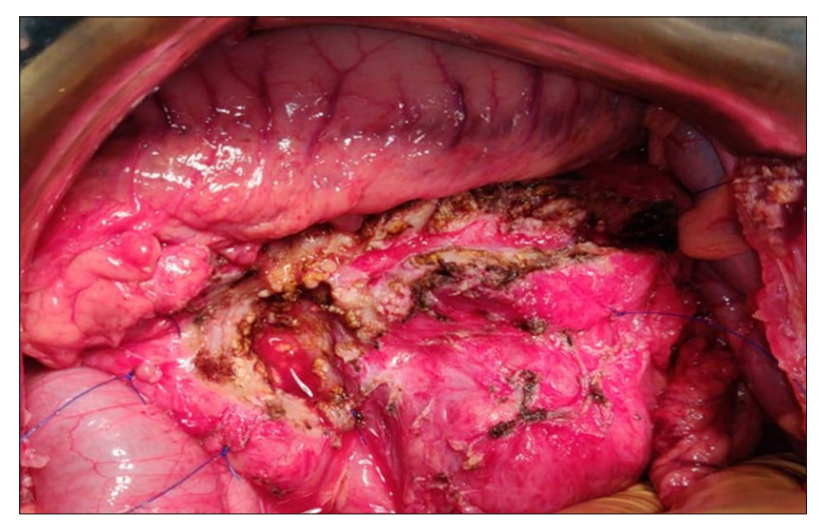

Fig. 3. Image showing completed bile-duct preserving pancreatic head resection (BDPPHR) technique. This image also highlights the possibility of leaving significant amount of pancreatic tissue by performing pancreatic head resection when posterior wall of pancreatic duct is considered as the limit of resection.

preserve pancreatic parenchyma in the superolateral aspect of head resection to safeguard the intrapancreatic portion of bile duct from injury. The superior and inferior extent of resection is limited by sparing $5 \mathrm{~mm}$ of thickness of pancreatic parenchyma in order to facilitate further pancreaticojejunostomy suturing. The medial extent i.e., left lateral extent of resection is highlighted by sparing a 5 $\mathrm{mm}$ rim of uncinate tissue along the medial wall of superior mesenteric vein to facilitate for placement of sutures during pancreaticojejunostomy and also to avoid any inadvertent injury to SMV during head resection. Rest of the medial extent of resection is limited by pancreatic duct in the neck region of pancreas. Posterior pancreatic capsule itself will be the posterior layer of pancreatic partition as shown in Figs. 3, 4. In this technique of BDPPHR, complete resection of pancreatic parenchyma in the head region of pancreas including the main and minor pancreatic ducts and uncinate ducts thoroughly with posterior pancreatic capsule as limit is performed.

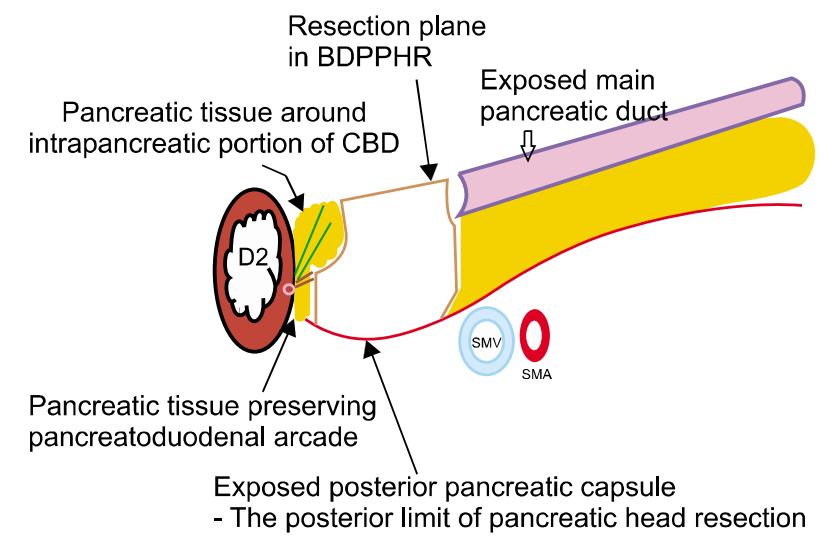

Fig. 4. Line diagram showing the view of pancreas from inferior aspect following BDPPHR with posterior pancreatic capsule as the posterior limit of pancreatic head resection.

The jejunum is divided at $25 \mathrm{~cm}$ distal to the duodenojejunal flexure and Roux limb is created. The Roux limb is passed through the mesocolic route to accommodate freely over the entire pancreas. A single layered pancreaticojejunostomy is usually performed using running 3-0 non-absorbable suture approximating jejunal mucosa to the capsule of the pancreas, instead of the mucosa of pancreatic duct, to facilitate drainage of small pancreatic ducts. The continuity of gastrointestinal tract is restored by side-to-side jejunojejunostomy in isoperistaltic fashion at $40 \mathrm{~cm}$ distal to pancreaticojejunostomy. All the mesenteric defects are closed with 3-0 non-absorbable sutures to prevent internal hernia formation. A $24 \mathrm{Fr}$ drain is routinely placed near the pancreaticojejunostomy site. Abdomen is closed in layers with continuous 1-0 absorbable sutures for inner layer and 1-0 non-absorbable sutures for outer layer.

\section{DISCUSSION}

Surgical management will be required in about $40-75 \%$ of patients with chronic pancreatitis during the course of their disease. ${ }^{4}$ A systematic review by Yang et al. ${ }^{5}$ highlighted that early surgical management for pain in chronic pancreatitis was associated with improved postoperative pain relief and reduced risk of pancreatic insufficiency. Currently on-going ESCAPE trial by Dutch study group will provide more information on importance of early surgical management for chronic pancreatitis. ${ }^{6}$ Among the resection procedures described for management of chronic 
pancreatitis, Frey's procedure is the most widely accepted. The defined extent of pancreatic head resection to be performed in Frey's procedure is still debatable. In his initial description of surgical procedure in 1987, Frey and Smith ${ }^{1}$ proposed that a shell or rim of pancreas should remain in the posterior aspect between the cored-out head and uncinate process and the inferior vena cava. He conceptualised that this shell of pancreatic tissue will prevent egress of pancreatic secretions into retroperitoneum. The exact extent of pancreatic tissue to be retained was not defined. He also proposed to expose the anterior surface of the intrapancreatic common bile duct in order to relieve constriction by fibrosis, which may not be the required scenario always. ${ }^{1}$ Frey and Amikura ${ }^{2}$ reported the results of the proposed Frey's procedure in 1994 after follow-up of 37 months. In this article, Frey and Amikura ${ }^{2}$ described that local resection of head of pancreas is achieved by excising the head of the pancreas overlaying the ducts of Wirsung and Santorini, and duct to the uncinate, along with their tributary ducts. The extent of resection described by Frey and Amikura ${ }^{2}$ in 1994 study has been assumed by many as modification of Frey's procedure by himself to minimise the risk of penetration of posterior pancreatic capsule. This varied description of the procedure has created some confusion to conclude the limit of posterior extent of pancreatic resection. Many further modifications of the proposed procedure of local resection of head of pancreas have been described in the literature.

Tan et al. ${ }^{7}$ conducted a retrospective review to compare the outcomes of original and modified Frey's procedure. He concluded that original Frey's procedure, inspite of being safe, achieved better pain relief and equal preservation of pancreatic function as compared to the modified Frey's procedure. Sakata et al reported technique of modified "minimum Frey's procedure" involving limited coring of pancreatic head to include only the area anterior to the main pancreatic duct. His findings stated that "minimum Frey's procedure" is sufficient for resolving intractable pain and improving nutritional status in most patients with chronic pancreatitis. ${ }^{3}$

Gloor et al. $^{8}$ reported a modified technique of Beger and Frey procedure in which retropancreatic dissection and transection of the pancreas is avoided in contrast to Beger procedure. In contrast to Frey's procedure, Gloor et al. ${ }^{8}$ advocated deliberate opening of common bile duct in intrapancreatic portion. In the present report of BDPPHR, pancreatic tissue in the supero-medial aspect of pancreatic head near the bile duct intra-pancreatic portion is preserved. Therefore, bile duct injury is avoided in the technique of BDPPHR. The posterior limit of pancreatic dissection was mentioned in the report by Gloor et al. ${ }^{8}$ as leaving behind a thin bridge of pancreatic tissue, similar to original Frey article. The exact extent of remnant pancreatic tissue after pancreatic head excision is definitely a matter of concern. Hence, the present report of BDPPHR is contemplated to accurately define the posterior limit of pancreatic head excision and also to alleviate concerns about the remnant pancreatic tissue in the head region.

In the present report, the posterior pancreatic capsule is considered as the posterior limit of pancreatic head resection. The authors wanted to reiterate the finding of thickened posterior pancreatic capsule observed in the patients of chronic pancreatitis during surgery. In order to avoid any inadvertent injury to posterior pancreatic capsule during kocherisation of duodenum, the authors recommend the plane of dissection to be close enough to inferior vena cava rather than to the posterior pancreatic capsule. In BDPPHR technique, perpendicular plane of pancreatic head excision is advised instead of saucerization of pancreatic head as shown in Fig. 2. This approach is facilitated by blunt dissection through the pancreatic tissue till the posterior pancreatic capsule is reached. Once the posterior pancreatic capsule is identified, slices of pancreatic tissue held with tissue holding forceps can be easily cleared from the pancreatic capsule

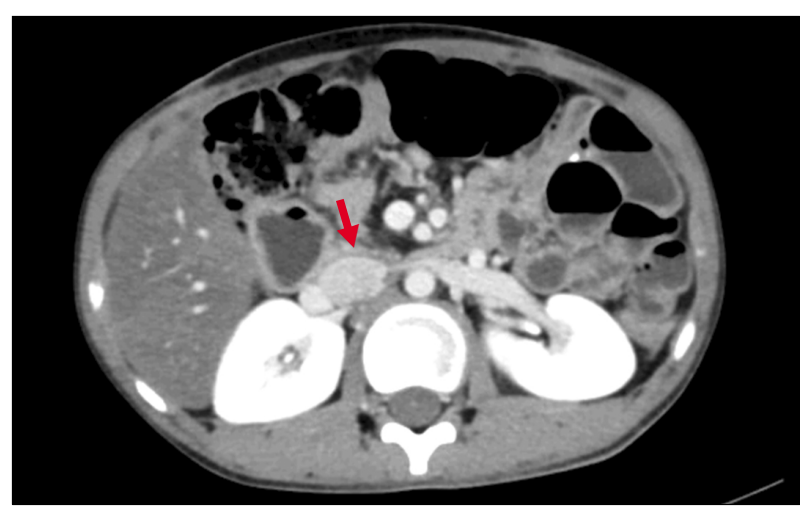

Fig. 5. CECT abdomen image of postoperative patient - six months following bile duct preserving pancreatic head resection with posterior pancreatic capsule as the limit of resection (pointed with red arrow). 
using even monopolar electrocoagulation applied at an angle parallel to posterior pancreatic capsule. This technique does not require utilisation of an ultrasonic aspirator and dissector as described by Andersen and Topazian ${ }^{9}$ for his technique of pancreatic head excavation. In the event of any breach in the posterior pancreatic capsule, re-approximation of the capsule with interrupted monofilament non-absorbable sutures is enough. In the author's experience, ten patients of chronic pancreatitis underwent the described technique of BDPPHR and none had any breach in the posterior pancreatic capsule during pancreatic head excision. None of the patients had developed pancreatic fistula during the postoperative period and in further follow up. During the follow up, none of the patients required admission for pain related to chronic pancreatitis. Among these 10 patients, three patients were known to have diabetes mellitus preoperatively. None of the patients developed new-onset diabetes in the post-operative follow up of one year. However, much longer follow up is required to assess the accurate efficacy of the procedure, especially regarding functional outcomes. Post-operative CECT image of patient following BDPPHR at six months follow up is shown in Fig. 5.

\section{CONCLUSION}

Therefore, BDPPHR is a safe, feasible and easily reproducible technique of pancreatic head excision. This technique can be accomplished with widely available monopolar electrocoagulation itself. The authors wanted to recapitulate the practicability of BDPPHR technique with the posterior pancreatic capsule as the limit of resection.

\section{ORCID}

Pagadala Naga Balaji Nitesh: https://orcid.org/0000-0002-9522-7149

Biju Pottakkat: https://orcid.org/0000-0002-8474-0270

\section{AUTHOR CONTRIBUTIONS}

Conceptualization: BP. Data curation: PNBN, BP. Formal analysis: PNBN, BP. Methodology: PNBN, BP. Project administration: BP. Visualization: PNBN, BP. Writing - original draft: PNBN, BP. Writing - review \& amp; editing: PNBN, BP.

\section{REFERENCES}

1. Frey CF, Smith GJ. Description and rationale of a new operation for chronic pancreatitis. Pancreas 1987;2:701-707.

2. Frey CF, Amikura K. Local resection of the head of the pancreas combined with longitudinal pancreaticojejunostomy in the management of patients with chronic pancreatitis. Ann Surg 1994; 220:492-504; discussion 504-507.

3. Sakata N, Egawa S, Motoi F, Goto M, Matsuno S, Katayose Y, et al. How much of the pancreatic head should we resect in Frey's procedure? Surg Today 2009;39:120-127.

4. Issa Y, van Santvoort HC, van Goor H, Cahen DL, Bruno MJ, Boermeester MA. Surgical and endoscopic treatment of pain in chronic pancreatitis: a multidisciplinary update. Dig Surg 2013; 30:35-50.

5. Yang CJ, Bliss LA, Schapira EF, Freedman SD, Ng SC, Windsor JA, et al. Systematic review of early surgery for chronic pancreatitis: impact on pain, pancreatic function, and re-intervention. J Gastrointest Surg 2014;18:1863-1869.

6. Ahmed Ali U, Issa Y, Bruno MJ, van Goor H, van Santvoort $\mathrm{H}$, Busch OR, et al. Early surgery versus optimal current step-up practice for chronic pancreatitis (ESCAPE): design and rationale of a randomized trial. BMC Gastroenterol 2013;13:49.

7. Tan CL, Zhang H, Yang M, Li SJ, Liu XB, Li KZ. Role of original and modified Frey's procedures in chronic pancreatitis. World J Gastroenterol 2016;22:10415-10423.

8. Gloor B, Friess H, Uhl W, Büchler MW. A modified technique of the Beger and Frey procedure in patients with chronic pancreatitis. Dig Surg 2001;18:21-25.

9. Andersen DK, Topazian MD. Pancreatic head excavation: a variation on the theme of duodenum-preserving pancreatic head resection. Arch Surg 2004;139:375-379. 